\title{
The anti-fibrotic agent SMP-534 attenuates bleomycin-induced pulmonary fibrosis in hamsters
}

\author{
Tsuyoshi Tsujimura ${ }^{1}$, Michiko Ono-Kishino ${ }^{1}$, Jun Nagamine ${ }^{1}$, Eiji Sugaru', Teruhisa Tokunaga ${ }^{2}$, Makoto \\ $\mathrm{KitOH}^{3}$, Ryu Nagata ${ }^{2}$, Tsutomu NaKagawa ${ }^{1}$ and Mutsuo TaiJi ${ }^{1}$ \\ ${ }^{1}$ Pharmacology Research Laboratories, ${ }^{2}$ Chemistry Research Laboratories and ${ }^{3}$ Process Chemistry Research \& Development Laborato- \\ ries, Dainippon Sumitomo Pharma Co., Ltd, Osaka, Japan \\ (Received 3 March 2009; and accepted 22 April 2009)
}

\begin{abstract}
Pulmonary fibrosis is a progressive and lethal lung disease characterized by accumulation of ECM and loss of pulmonary function. However, no cure exists for this disease, and current treatments often fail to slow its progression or relieve its symptoms. We have previously reported that the anti-fibrotic agent SMP-534 has beneficial effects on renal fibrosis in animal model of nephropathy. In this study, we examined whether SMP-534 has beneficial effects on pulmonary fibrosis in bleomycin-treated hamsters. Treatment with SMP-534 [low dose $(70 \mathrm{mg} / \mathrm{kg})$ or high dose $(110 \mathrm{mg} / \mathrm{kg})$ ] counteracted inhibition of body weight increase induced by bleomycin. In addition, SMP-534 significantly inhibited bleomycin-induced increase in lung hydroxyproline level, an index of collagen formation. Moreover, SMP-534 significantly ameliorated histological pulmonary fibrotic changes induced by bleomycin. The results of this study indicate that the anti-fibrotic agent SMP-534 may offer a new therapeutic option for the treatment of pulmonary fibrosis.
\end{abstract}

Pulmonary fibrosis is a condition initiated by acute or chronic lung injury and is characterized by deposition of extracellular matrixes (ECM) in the lung (4). In idiopathic pulmonary fibrosis, pulmonary function progressively deteriorates, resulting in high mortality rate $(3,16)$. Treatment options for patients with idiopathic pulmonary fibrosis are very limited as clinical data has demonstrated that current therapies often fail to delay progression of the disease or relieve symptoms $(6,16)$. Therefore, new agents that effectively act on pulmonary fibrosis have been waited.

Previous studies have demonstrated that TGF- $\beta$ is a key factor in the initiation and development of pulmonary fibrosis in human and animals $(2,9)$.

Address correspondence to: Tsutomu Nakagawa Ph.D. Discovery Pharmacology I, Pharmacology Research Laboratories, Dainippon Sumitomo Pharma Co., Ltd., 3-1-98 Kasugadenaka, Konohana-ku, Osaka 554-0022, Japan Tel: +81-6-6466-5299, Fax: +81-6-6466-5491

E-mail: tsutomu-nakagawa@ds-pharma.co.jp
TGF- $\beta$ promotes accumulation of ECM by stimulating transcription of collagen genes and by inhibiting transcription of collagenase. Additionally, TGF- $\beta$ levels have been found to correlate with the severity of lung function abnormalities, lung pathology, and reduced survival time in human (8). These results suggest that TGF- $\beta$ is a good drug target for pulmonary fibrosis.

We have previously demonstrated that the anti-fibrotic agent SMP-534 inhibits ECM accumulation induced by TGF- $\beta$ in fibroblasts through inhibition of TGF- $\beta$ signaling cascade (12). Additionally, we have studied whether SMP-534 has beneficial effects on renal fibrosis. We have shown that treatment with SMP-534 inhibits accumulation of ECM in kidney of diabetic $d b / d b$ mouse or $5 / 6$ nephrectomized rats and ameliorates renal function $(13,15)$. In the present study, we investigated whether SMP-534 has beneficial effects on pulmonary fibrosis in bleomycin-treated hamsters. 


\section{MATERIALS AND METHODS}

Chemicals. SMP-534 was synthesized in our laboratories. Bleomycin (Sigma-ALDRICH, Inc.) was commercially purchased.

Animals. Male hamsters housed in a temperatureand humidity-controlled room $\left(21 \sim 27^{\circ} \mathrm{C} / 40 \sim 70 \%\right)$ under a 12-hour light/dark cycle were used in this study. The animals had free access to diet (powder chow), with or without drug, and tap water throughout the experimental period. All procedures were approved by Dainippon Sumitomo Pharmaceutical Committee on Animal Research.

Study design. Male hamsters were purchased from Japan SLC, Inc. (Shizuoka, Japan). The animals were randomly divided into four groups [saline + vehicle group $(\mathrm{n}=8)$, bleomycin + vehicle group $(\mathrm{n}=9)$, bleomycin + SMP-534 (low dose) group $(\mathrm{n}=9)$, and bleomycin + SMP-534 (high dose) group $(\mathrm{n}=9)$ ]. SMP-534 was admixed with powder chow (Rodent Laboratory Chow 5001; Purina Mills Inc. USA) and administered to hamsters at $0.1 \%$ (low dose, about $70 \mathrm{mg} / \mathrm{kg}$ ) or $0.2 \%$ (high dose, about $110 \mathrm{mg} / \mathrm{kg}$ ) for 4 weeks. Two days after SMP-534 administration, the hamsters were intratracheally treated once with saline or bleomycin $(10 \mathrm{U} / \mathrm{kg})$. Body weight was measured for 4 weeks after bleomycin administration, and the development of pulmonary fibrosis was assessed at 4 weeks after bleomycin administration.

Measurement of lung hydroxyproline content. Hamsters were killed under anesthesia at 4 weeks after bleomycin administration and the left lungs were removed. Frozen lung tissues were homogenized by a polytron tissue homogenizer in PBS and dried. The pellets were hydrolyzed for $3 \mathrm{~h}$ at $100^{\circ} \mathrm{C}$ in $4 \mathrm{~N}$ $\mathrm{NaOH}$. Hydroxyproline in the hydrolysate was assessed colorimetrically at $550 \mathrm{~nm}$ with p-dimethylaminobenzaldehyde $(7,17)$.

Lung histological analysis. Hamsters were killed under anesthesia at 4 weeks after bleomycin administration, and the right lung was fixed with $10 \%$ buffered formalin and embedded in paraffin. Tissue slices were stained with hematoxylin-eosin and Masson-trichrome, and fibrosis score was semiquantitatively assessed according to the method of Ashcroft et al. (1).

Statistical analysis. Data are presented as the mean \pm SD. Differences between individual groups were analyzed by Student's $t$-test. Fibrosis score was compared between individual groups using nonparametric analysis based on Wilcoxon test. In all cases, $P<0.05$ was considered to be statistically significant.

\section{RESULTS}

\section{Effect of SMP-534 on body weight}

Treatment with bleomycin significantly inhibited increase in body weight of hamsters as compared to treatment with saline. However, increase in body weight in the bleomycin + SMP-534 (low or high dose)-treated groups was not different from that in the saline + vehicle-treated group (Fig. 1 A, B).

\section{Effect of SMP-534 on hydroxyproline content in the lung}

As an index of collagen formation, lung hydroxyproline content was measured to evaluate the effect of SMP-534 on ECM accumulation in the lung. Hydroxyproline content in the bleomycin + vehicletreated group was $176 \%$ that in the saline + vehicletreated group. Treatment with the high dose SMP-534 significantly inhibited bleomycin-induced increase in hydroxyproline level, although the low dose of SMP-534 had no significant effect on lung hydroxyproline level (Fig. 2).

\section{Lung histological analysis}

To investigate lung histological and fibrotic changes induced by bleomycin and the effects of SMP-534 on these changes, hematoxylin-eosin staining and Masson-trichrome staining of the lung were performed at the end of the study period. We observed from the analysis of hematoxylin-eosin staining that treatment with bleomycin led to significant pulmonary histological changes, including thickening of the alveolar walls and interstitial tissue (Fig. 3). Additionally, we observed from the analysis of Masson-trichrome staining that treatment with bleomycin induced significant ECM accumulation in interstitial tissue (Fig. 4). However, treatment with high dose SMP-534 inhibited pulmonary histological changes and reduced ECM accumulation in interstitial tissue (Figs. 3, 4).

To semiquantitatively evaluate bleomycin-induced pulmonary fibrosis, fibrotic changes were scored according to the method of Ashcroft et al. (1). Ashcroft method is a standard method used to evaluate the severity of pulmonary fibrosis by assigning a score $(0 \sim 8)$ to lung histological changes, such as 
(A)

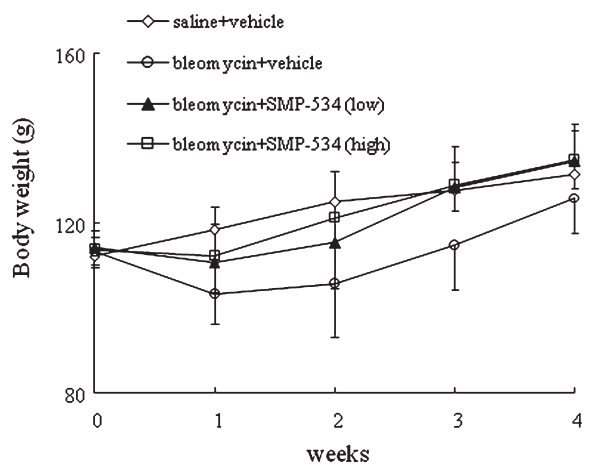

(B)

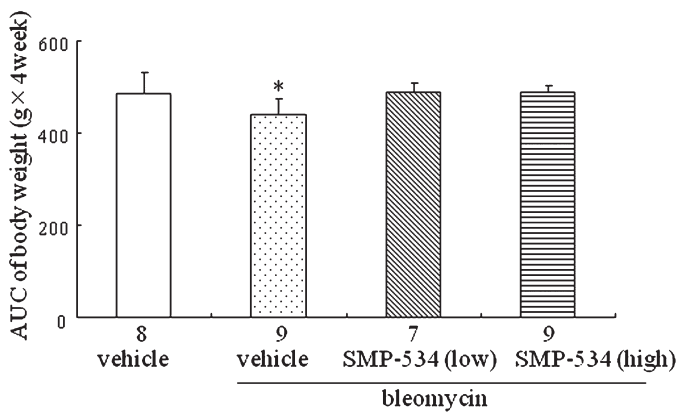

Fig. 1 Effect of SMP-534 on body weight in hamsters. Body weight was measured from 0 to 4 weeks after bleomycin administration. (A) Change in body weight and (B) Area under increase of body weight curve during study period. Data are presented as the mean \pm SD. ${ }^{*} P<0.05$ vs the saline + vehicle-treated group (Student's $t$-test). During the study period, two hamsters out of nine in the low dose SMP-534-treated group died, whereas all hamsters in other groups survived.

thickening of alveolar or bronchiolar walls and damaged lung architecture. In the bleomycin-treated group, fibrosis score was high in all lobes (anterior, medial, and posterior) of the lung. However, in the hamsters treated with the high dose of SMP-534, fibrosis score in the lung medial or posterior lobe was significantly lower than that in the bleomycin-treated group (Fig. 5).

\section{DISCUSSION}

The aim of this study was to investigate whether the anti-fibrotic agent SMP-534 has beneficial effects on pulmonary fibrosis. We found for the first time that treatment with SMP-534 inhibits the progression of pulmonary fibrosis in bleomycin-treated hamsters. Our results show that treatment with SMP-534 counteracted inhibition of body weight increase induced by bleomycin. In addition, SMP-534 significantly inhibited bleomycin-induced increase in lung hydroxyproline level, an index of collagen formation. Moreover, SMP-534 significantly ameliorated

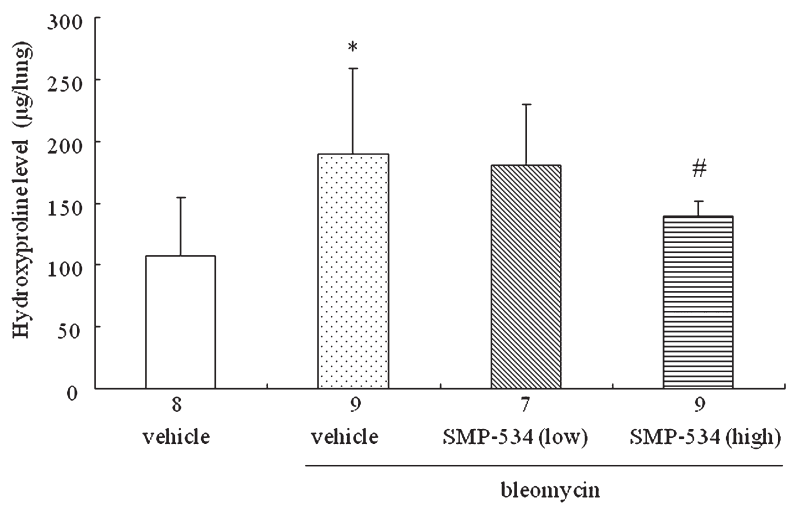

Fig. 2 Effect of SMP-534 on hydroxyproline content in the lung. Hydroxyproline content in the lung was measured at 4 weeks after bleomycin administration. Data are presented as the mean $\pm \mathrm{SD}$. ${ }^{*} P<0.05$ vs the saline + vehicle-treated group (Student's $t$-test). \#P<0.05 vs the bleomycin + vehicletreated group (Student's $t$-test). Numbers in the $X$ axis indicate survival hamsters.

histological pulmonary fibrotic changes induced by bleomycin.

Pulmonary fibrosis is a progressive and lethal lung disease (4). Idiopathic pulmonary fibrosis is the most common type of pulmonary fibrosis, leading to a high incidence of death (5 year mortality rate: $>$ $50 \%$ ) (3). Corticosteroids and immunosuppressive agents are generally used for the treatment of idiopathic pulmonary fibrosis $(6,16)$. However, it is known that the beneficial effects of these agents are very poor $(6,16)$. Recently, a clinical study has shown that treatment with pirfenidone, a new agent for idiopathic pulmonary fibrosis, can ameliorate pulmonary function in patients with idiopathic pulmonary fibrosis. Accordingly, pirfenidone is now used for the treatment of idiopathic pulmonary fibrosis in Japan. It is known that pirfenidone induces its anti-fibrotic effect by decreasing the expression of TGF- $\beta$ (14). On the other hands, we have previously demonstrated that the anti-fibrotic effect of SMP-534 is produced via inhibition of TGF- $\beta$ signaling cascade (12). Therefore, the mechanism by which SMP-534 acts on idiopathic pulmonary fibrosis is different from that of pirfenidone, allowing a new therapy for the disease. Additionally, it is believed that combination therapy with SMP-534 and pirfenidone might be more effective for the treatment of idiopathic pulmonary fibrosis than the therapy with pirfenidone alone.

Many reports have shown that TGF- $\beta$ is important for the initiation and development of pulmonary fibrosis $(2,9)$. Therefore, TGF- $\beta$ has been thought to be a good drug target of pulmonary fibrosis. Un- 


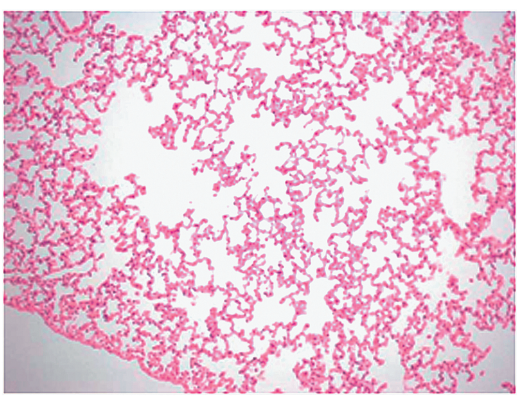

saline + vehicle

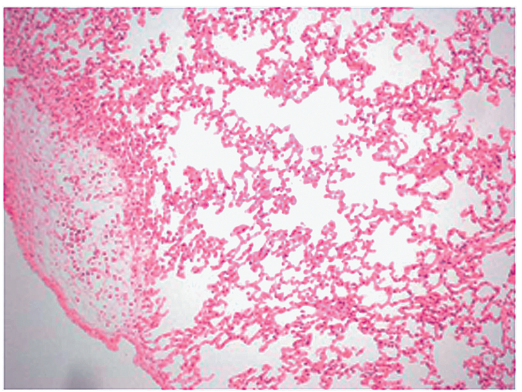

bleomycin + SMP-534 (low)

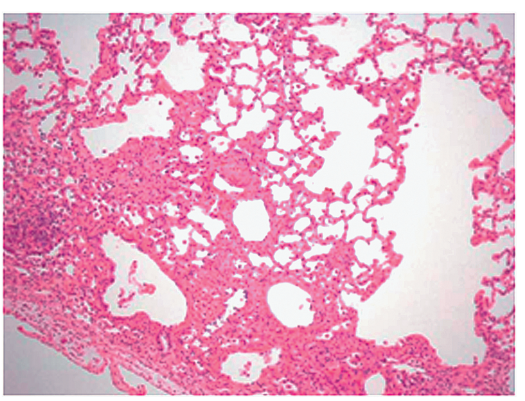

bleomycin + vehicle

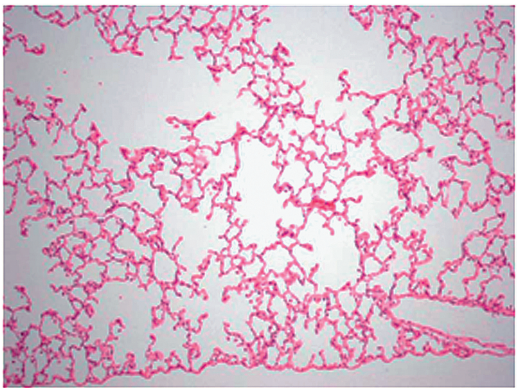

bleomycin + SMP-534 (high)

Fig. 3 Effect of SMP-534 on bleomycin-induced lung histopathologic alteration. Representative photomicrographs of hematoxylin-eosin stained lung sections of posterior lobe from saline + vehicle-treated hamsters, bleomycin + vehicle-treated hamsters, bleomycin + SMP-534 (low dose)-treated hamsters or bleomycin + SMP-534 (high dose)-treated hamsters (Original magnification $\times 100)$.

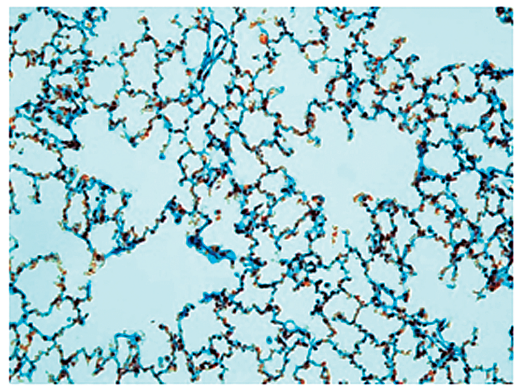

saline + vehicle

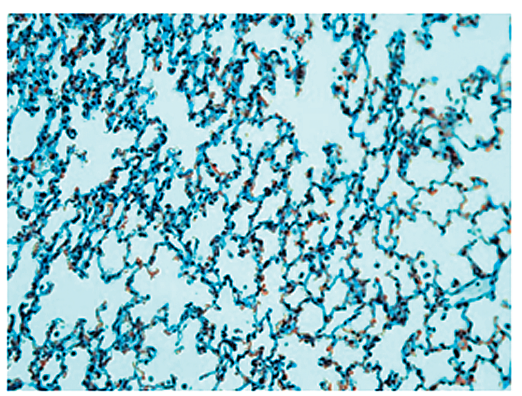

bleomycin + SMP-534 (low)

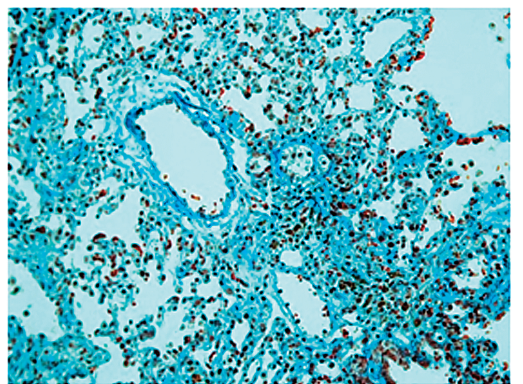

bleomycin + vehicle

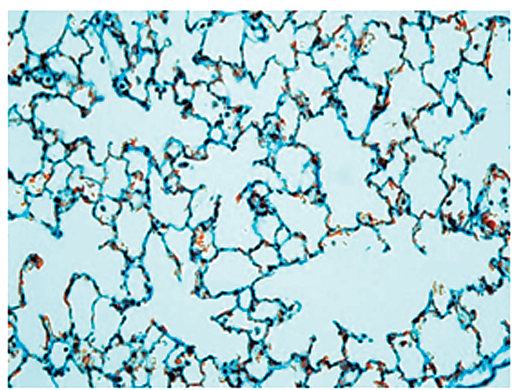

bleomycin + SMP-534 (high)

Fig. 4 Effect of SMP-534 on bleomycin-induced lung ECM accumulation. Representative photomicrographs of Massontrichrome stained lung sections of posterior lobe from saline + vehicle-treated hamsters, bleomycin + vehicle-treated hamsters, bleomycin + SMP-534 (low dose)-treated hamsters or bleomycin + SMP-534 (high dose)-treated hamsters (Original magnification $\times 400$ ). 
Anterior lobe

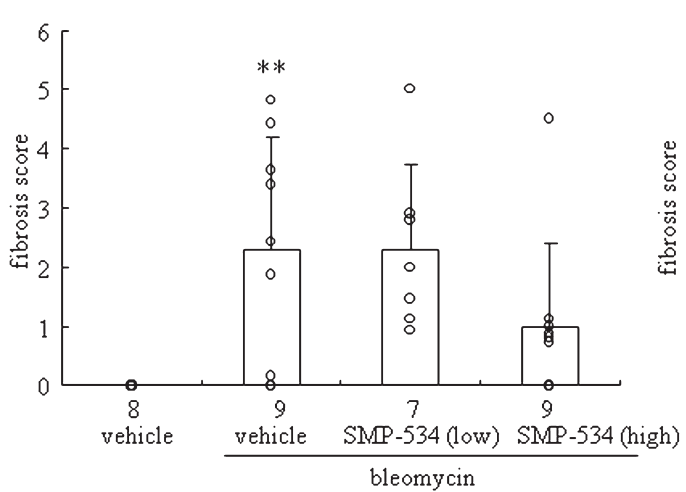

Medial lobe

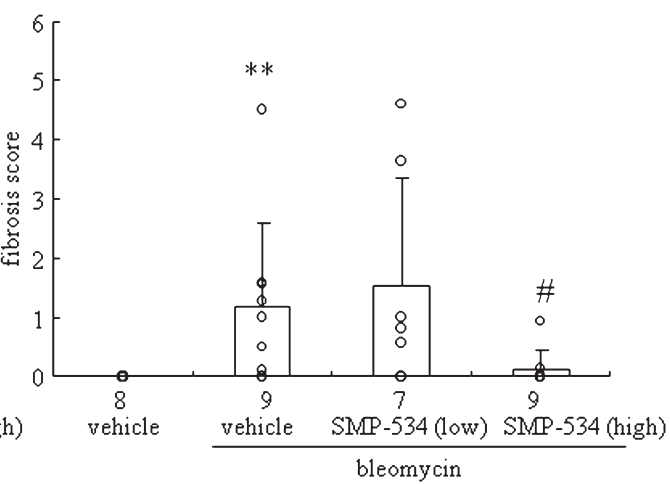

Posteriorlobe

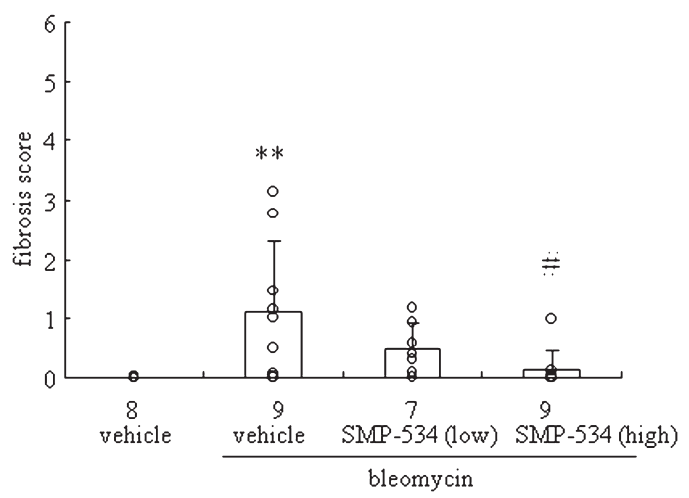

Fig. 5 Effect of SMP-534 on lung fibrosis score. Expansion of pulmonary fibrosis in the anterior, medial, or posterior lobe was scored according to the method of Ashcroft et al. Data are presented as the mean $\pm \mathrm{SD}$. ${ }^{* *} P<0.01$ vs the saline + vehicle-treated group (Wilcoxon test). $\# P<0.05$ vs the bleomycin + vehicle-treated group (Wilcoxon test). Numbers in the $X$ axis and circle $(\mathrm{O})$ in the figure indicate survival hamsters.

til now, it has been reported that TGF- $\beta$ antibody is effective in inhibiting the progression of pulmonary fibrosis in bleomycin-treated mice (5). However, we believe that inhibition of all TGF- $\beta$ signaling cascade by TGF- $\beta$ antibody might induce unexpected side effects, because TGF- $\beta$ is a multifunctional cytokine involving growth, differentiation, and immunosuppression $(10,11)$. Therefore, we tried to find a novel compound that inhibits TGF- $\beta$ signaling cascade related to ECM accumulation only, and finally obtained SMP-534. We expect that SMP-534 will be a new therapeutic agent for the treatment of pulmonary fibrosis.

In this study, we have shown that SMP-534 has beneficial effects on pulmonary fibrosis in hamsters. Treatment with SMP-534 was started concomitantly with the progression of pulmonary fibrosis induced by bleomycin, demonstrating the efficacy of SMP-534 treatment in prevention protocol. Therefore, in the future, it would be interesting to examine whether SMP-534 has similar beneficial effects on an already- established pulmonary fibrotic damage. In addition, it is very important to evaluate the effects of SMP-534 on other organs fibrosis, such as liver cirrhosis, scleroderma, and cardiovascular fibrosis since effective treatment for all these diseases is highly needed.

\section{REFERENCES}

1. Ashcroft T, Simpson JM and Timbrell V (1988) Simple method of estimating severity of pulmonary fibrosis on a numerical scale. J Clin Pathol 41, 467-470.

2. Bartram U and Speer CP (2004) The role of transforming growth factor beta in lung development and disease. Chest 125, 754-765.

3. Coultas DB, Zumwalt RE, Black WC and Sobonya RE (1994) The epidemiology of interstitial lung diseases. Am J Respir Crit Care Med 150, 967-972.

4. Crouch E (1990) Pathobiology of pulmonary fibrosis. Am J Physiol 259, L159-L184.

5. Giri SN, Hyde DM and Hollinger MA (1993) Effect of antibody to transforming growth factor beta on bleomycin induced accumulation of lung collagen in mice. Thorax 48, 959-966. 
6. Gross TJ and Hunninghake GW (2001) Idiopathic pulmonary fibrosis. $N$ Engl J Med 345, 517-525.

7. Huszar G, Maiocco J and Naftolin F (1980) Monitoring of collagen and collagen fragments in chromatography of protein mixtures. Anal Biochem 105, 424-429.

8. Khalil N, O'Connor RN, Unruh HW, Warren PW, Flanders KC, Kemp A, Bereznay OH and Greenberg AH (1991) Increased production and immunohistochemical localization of transforming growth factor-beta in idiopathic pulmonary fibrosis. Am J Respir Cell Mol Biol 5, 155-162.

9. Krein PM and Winston BW (2002) Roles for insulin-like growth factor I and transforming growth factor-beta in fibrotic lung disease. Chest 122, 289S-293S.

10. Letterio JJ and Roberts AB (1998) Regulation of immune responses by TGF-beta. Annu Rev Immunol 16, 137-161.

11. Moustakas A, Pardali K, Gaal A and Heldin CH (2002) Mechanisms of TGF-beta signaling in regulation of cell growth and differentiation. Immunol Lett 82, 85-91.

12. Sugaru E, Sakai M, Horigome K, Tokunaga T, Kitoh M, Hume WE, Nagata R, Nakagawa $T$ and Taiji $M$ (2005) SMP-534 inhibits TGF-beta-induced ECM production in fibroblast cells and reduces mesangial matrix accumulation in experimental glomerulonephritis. Am J Physiol Renal Physiol
289, F998-F1004.

13 Sugaru E, Nakagawa T, Ono-Kishino M, Nagamine J, Tokunaga T, Kitoh M, Hume WE, Nagata R and Taiji M (2006) SMP-534 ameliorates progression of glomerular fibrosis and urinary albumin in diabetic $d b / d b$ mice. Am J Physiol Renal Physiol 290, F813-F820.

14. Tian XL, Yao W, Guo ZJ, Gu L and Zhu YJ (2006) Low dose pirfenidone suppresses transforming growth factor beta- 1 and tissue inhibitor of metalloproteinase-1, and protects rats from lung fibrosis induced by bleomycina. Chin Med Sci J 21, 145-151.

15. Tsujimura T, Nagamine J, Sugaru E, Nakagawa T, OnoKishino M, Tokunaga T, Kitoh M, Nagata R and Taiji M (2008) Chronic administration of SMP-534 ameliorates renal dysfunction in 5/6 nephrectomized rats. Nephron Exp Nephrol 110, e99-e108.

16. White ES, Lazar MH and Thannickal VJ (2003) Pathogenetic mechanisms in usual interstitial pneumonia/idiopathic pulmonary fibrosis. J Pathol 201, 343-354.

17. Woessner JF, Jr (1961) The determination of hydroxyproline in tissue and protein samples containing small proportions of this imino acid. Arch Biochem Biophys 93, 440-447. 